\title{
Convolutional Neural Network for Classification of Eight types of Arrhythmia using 2D Time-Frequency Feature Map from Standard 12-Lead Electrocardiogram
}

Da Un Jeong

Kumoh National Institute of Technology

Ki Moo Lim ( $\sim$ kmlim@kumoh.ac.kr)

Kumoh National Institute of Technology

\section{Research Article}

Keywords: Neural Network, Electrocardiogram, CNN, STFT

Posted Date: April 8th, 2021

DOI: https://doi.org/10.21203/rs.3.rs-396762/v1

License: (a) (1) This work is licensed under a Creative Commons Attribution 4.0 International License. Read Full License 


\section{Abstract}

Electrocardiograms (ECGs) are widely used for diagnosing cardiac arrhythmia based on the deformation of signal shapes due to changes in various heart diseases. However, these abnormal signs may not be observed in some of the 12 ECG channels, depending on the location or shape of the heart and the type of cardiac arrhythmia. Therefore, to accurately diagnose cardiac arrhythmias, it is necessary to closely and comprehensively observe ECG signals acquired from 12 channel electrodes. In this study, we proposed a clustering algorithm that can classify persistent cardiac arrhythmia as well as episodic cardiac arrhythmias using the standard 12-lead ECG signals and the 2D CNN model using the timefrequency feature maps to classify the eight types of arrhythmias including normal sinus rhythm. The standard 12-lead ECG dataset was provided by Computing in Cardiology 2020 Physionet Challenge and consisted of 6,877 patients. The proposed algorithm showed excellent performance in the classification of persistent cardiac arrhythmias; however, its accuracy was somewhat low in the classification of episodic arrhythmias. If our proposed model is trained and verified using more clinical data, we believe it can be used as an auxiliary device for diagnosing cardiac arrhythmias.

\section{Introduction}

Electrocardiograms (ECGs) are widely used for diagnosing cardiac arrhythmia based on the deformation of signal shapes due to changes in the electrical and mechanical activity of the heart as a result of various heart diseases $[1,2]$. The standard 12-lead ECG records the electrical activity of the heart in four directions: inferior (lead II, III, and aVF), anterior (lead I, aVL, V1, and V2), septal (V3 and V4), and lateral (V5 and V6). Therefore, the shape of the signal in each channel varies according to the type of cardiac arrhythmia, and abnormal symptoms may be observed only in a specific channel among the 12 channels. Among these cardiac arrhythmias, abnormal symptoms are continuously observed on the ECG as persistent symptoms. Some symptoms are also observed intermittently with normal signals [2]. Therefore, it is not easy to detect cardiac arrhythmia by looking at the ECG signal with the naked eye. To accurately diagnose cardiac arrhythmias, it is necessary to closely and comprehensively observe ECG signals acquired from 12 channel electrodes.

In this study, cardiac arrhythmias in which abnormal signs are continuously observed in the ECG signal were defined as 'persistent cardiac arrhythmias' (the meaning of 'persistent' in 'persistent cardiac arrhythmias' is different from that in 'persistent atrial fibrillation'). Representative persistent cardiac arrhythmias include atrial fibrillation (AF), first-degree atrioventricular block (I-AVB), left bundle branch block (LBBB), and right bundle branch block (RBBB). In the ECG signal of AF patients, an intact shape of the QRS wave is observed; however, because of the multiple irregular electrical simulations generated in the atrium, the $P$ wave does not appear, and irregularly oscillating fibrillatory waves are observed [3]. In the case of I-AVB, the PR interval was continuously observed to be longer than $200 \mathrm{~ms}$ compared to that observed for a normal heart [2, 4]. In the case of LBBB and RBBB, abnormal signs consistently occur, but they do not occcur on all of the 12 channels. In the LBBB condition, an M-shaped signal is often observed 
However, it is not easy to distinguish abnormal signs of persistent cardiac arrhythmia because of the presence of motion artifacts, noise due to contact with electrodes, and noise produced by the measuring device itself during the acquisition of the ECG signal.

Meanwhile, unlike persistent cardiac arrhythmias, cardiac arrhythmias that occur intermittently in normal signals are defined as "episodic cardiac arrhythmias." ST-segment elevation (STE) and ST-segment depression (STD), which are mainly caused by myocardial ischemia or infarction, are observed intermittently in the normal sinus rhythm (NSR). They are diagnosed when the height difference between the S-peak and the PR segment, which denotes the slope of the ST-segment of the ECG, is greater than \pm $0.5 \mathrm{~mm}$. The STD patient has an S-peak $3 \mathrm{~mm}$ lower than the PR segment in leads I, V5, V6, and aVL, and normal rhythms may appear in leads V2-V3 [6]. On the other hand, in the case of STE, an S-peak that is 4 $\mathrm{mm}$ higher than the PR segment is observed. In the ischemic ST segment, the ECG signal at V2-V4 is almost normal; however, in the case of the non-ischemic ST segment, abnormal symptoms are also observed at V2-V3 [2]. Premature atrial contraction (PAC) occurs when the atrial tissue is temporarily excited above the threshold more quickly than the sinus node, and it is a common symptom that $80 \%$ of people experience intermittently during their daily lives $[6,7]$. P-waves are not observed in the ECG signal of premature ventricular contraction (PVC) patients, and the heart rate is increased compared to that of a normal heart. In addition, the QRS width of PVC patients can increase by 120 to $210 \mathrm{~ms}$ compared to that of normal people, which is $60-80 \mathrm{~ms}[1,8]$. However, it is difficult to accurately diagnose such episodic cardiac arrhythmias because their abnormalities are intermittently observed during normal heart rhythm.

Many research groups have proposed computer-aided diagnosis algorithms for diagnosing cardiac arrhythmias using ECG signals [3, 7-9]. Inan et al. succeeded in classifying the PVC rhythm with $95.2 \%$ accuracy using a robust neural network after extracting the timing interval feature through wavelet transform to classify the beat of NSR and the abnormal rhythm due to PVC [8]. Shi et al. successfully detected the ST-segments in ECG signals using wavelet transform for the diagnosis of STD and STE [10]. In the 2017 Physionet challenge, which distinguished between normal, AF-induced signals, and other abnormal rhythms from ECG signals obtained from a short-single-lead ECG, Data et al. used short-time Fourier transform (STFT) to remove motion artifacts from ECG signals and achieved the highest classification performance (F1 score of 0.83 ) through multiple binary classifications [11]. In addition, Mehmood et al. classified cardiac arrhythmias through a two-dimensional (2D) ECG spectral image using STFT [9]. These algorithms classify cardiac arrhythmia by extracting the time-frequency features from a single ECG lead. However, as mentioned earlier, since some cardiac arrhythmias are observed only in a specific ECG channel, classification accuracy may be reduced based on the type of cardiac arrhythmia to be classified.

Some studies have classified cardiac arrhythmias based on standard 12-lead ECG records. Dohare et al. and Baloglu et al. classified several types of myocardial infarctions from 12-lead ECG signals using a support vector machine and 1D deep CNN structure $[12,13]$. They achieved high accuracies of $98.96 \%$ [12] and $99.7 \%$ [13], respectively. Ribeiro et al. classified six types of cardiac arrhythmias with F1 scores ch were composed of stacked transformations [14]. Mostayed 
et al. proposed a bidirectional LSTM network classifier to detect nine arrhythmias, including NSR, and achieved a final F1 score of 74.15 [15].

The aforementioned studies either focused on the characteristics of the ECG itself due to arrhythmias without distinguishing between persistent cardiac arrhythmias and episodic cardiac arrhythmias or classified specific arrhythmias using extracted features through a feature engineering process. Since persistent cardiac arrhythmia and episodic cardiac arrhythmia have different tendencies in ECG signals, it is necessary to extract features by considering each characteristic for an accurate diagnosis of cardiac arrhythmia.

In this study, we proposed an algorithm to automatically extract abnormalities due to episodic cardiac arrhythmias, including persistent cardiac arrhythmia, from standard 12-lead ECG signals to accurately detect cardiac arrhythmias in patients. The algorithm is composed of a convolutional neural network (CNN) using a 2D time-frequency feature map to classify the eight types of cardiac arrhythmia: AF, I-AVB, LBBB, RBBB, PAC, PVC, STD, and STE.

\section{Results}

The results of classifying eight cardiac arrhythmias using the proposed algorithm are shown in Fig. 3 . The F1 scores of the proposed model were over 0.8 , during the classification of persistent cardiac arrhythmias such as AF, I-AVB, LBBB, and RBBB. The highest F1 score was observed for LBBB prediction at 0.89 , and the classified $F 1$ scores for $A F, R B B B$, and I-AVB were $0.86,0.85$, and 0.80 , respectively. On the other hand, the classification performance of episodic cardiac arrhythmias such as PAC, PVC, and STE was relatively low compared to that of persistent cardiac arrhythmias. The lowest F1 score (0.52) was observed during the classification of STE, which had the smallest number of data classes. The F1 scores for PAC and PVC were 0.53 and 0.64 , respectively. In addition, the F1 scores for NSR and STD were moderate with scores of 0.77 and 0.76 , respectively. There was a difference in the number of data classes used for training and testing. Accordingly, the final macro F1 score of the proposed model was 0.74 ; however, the weighted F1 score, considering the difference in the number of data classes, was 0.78 .

Two performance curves were used to quantitatively evaluate the classification performance of the proposed model (Fig. 4). Receiver operating characteristic (ROC) curves show the true positive rate according to the false positive rate of the classified data, and the classification accuracy of each class can be evaluated using the area under the curve (AUC). The AUCs of the persistent cardiac arrhythmia classes were $0.90,0.90,0.91$, and 0.90 , respectively. In the episodic cardiac arrhythmia classes, the AUC of the STD was the highest at 0.89 , and that for the NSR was 0.89 . The other episodic cardiac arrhythmia classes showed moderate accuracies of 0.72 for PAC, 079 for PVC, and 0.78 for STE, respectively (Fig. 4a).

As mentioned earlier, the standard ECG dataset used in the model training and testing were different based on the number of data classes. Accordingly, the classification performance of the model was Loading [MathJax]/jax/output/CommonHTML/jax.js hile taking the dataset imbalance into account. The 
classification accuracy, after adjusting for the ratio of the data classes, showed excellent performance, with over 0.90 AUC for AF, LBBB, and RBBB among the persistent cardiac arrhythmia groups (AUCs: 0.93 AF, 0.94; I-AVB, 0.93; RBBB). The classification accuracies of I-AVB, NSR, and STD were over 0.80 (AUCs; 0.88 for I-AVB, 0.85 for NSR, and 0.82 for STD). However, as in the ROC curves, the AUCs for PAC, PVC, and STE were $0.52,0.68$, and 0.55 , respectively, showing a rather low classification accuracy (Fig. $4 \mathrm{~b}$ ).

\section{Discussion}

We proposed an automatic feature extraction algorithm and a CNN classification model for the classification of nine types of cardiac arrhythmias, including NSR. The main findings of this study are as follows:

1. The F1 score of the proposed model for arrhythmia classification was 0.78 .

2. In the performance curves, the AUC of the proposed model was over 0.90 in the classification of persistent cardiac arrhythmias (AF, LBBB, and RBBB). This means that our proposed model is more suitable for the classification of persistent cardiac arrhythmias than for the classification of episodic cardiac arrhythmias.

In this study, the STFT of ECG signals containing 2-3 PQRST waves was performed to detect the change in the dominant frequency with time. In the ECG waveform, the dominant frequency with the largest spectral power density can vary depending on the presence of heart disease, and abnormal symptoms due to cardiac arrhythmias can also be traced through frequency changes [16-19]. These abnormal signs due to cardiac arrhythmia could be observed in the ECG signals extracted through the clustering algorithm proposed in this study, and these were reflected in the time-frequency map generated during the STFT (Supplementary Figure S1-S11).

In AF patients, irregular waveforms are captured in the P-wave of the ECG, and the frequency during AF ranges from $5.3 \mathrm{~Hz}$ to $10 \mathrm{~Hz}$ [20]. This was also observed in the time-frequency map of the proposed algorithm in this study (Supplementary Figure S2). Our results were similar to those of Stridh et al. [18]. Furthermore, the change in the waveform due to LBBB and RBBB was captured in the ECG waveforms of leads I, V5, and V6 (LBBB), and leads III, aVL, and aVF (RBBB) and their time-frequency feature maps (Supplementary Figure S11-S14). These results are consistent with those of Benmalek et al. [21], whose study involved time-frequency spectrogram analysis.

PAC patients have an abnormal P-wave shape, which denotes the electrical depolarization of the atrium. Sometimes, this abnormal P-wave shape is partially or completely buried in the T-wave due to the electrical repolarization of the ventricles. However, in most PAC patients, either the interval between the Pwave and the QRS wave is slightly longer than that in the normal ECG or the P-wave and the QRS wave appear similar to the sinus rhythm, making it difficult to distinguish it from that of a normal heart [6]. These forms can also be confirmed in the ECG signals selected in this study using the proposed clustering algorithm (Supplementary Figure S7). On the other hand, in the case of STD and STE, the rise 
and fall of the ST segment was captured in V 5 and V6, and a more pronounced difference was observed in the temporal frequency maps of V5 and V6 (Supplementary Figure S15-S18).

In the standard 12-lead ECG dataset used in this study, signals with multiple labels were acquired from patients who had symptoms of two or more cardiac arrhythmias (Supplementary Table S1) [22]. Some PAC patients had abnormalities due to STD and STE, and in the signals of LBBB patients, the RBBB and AF signals were observed simultaneously. Accordingly, in situations where our proposed clustering algorithm was unable to extract the signal of the target arrhythmia but was able to extract the signal due to the complication as a representative signal, the error of the arrhythmia classification was able to increase. Furthermore, the classification error might have increased since the noisy signal was extracted as a representative signal rather than the most obvious signal in the NSR (Supplementary Figure S1). Nevertheless, the accuracy during the classification of persistent cardiac arrhythmias, such as AF, LBBB, and RBBB, was high.

In this study, the number of ECG datasets for each arrhythmia group was unbalanced. The arrhythmia group with the most dataset was RBBB $(1,675)$, excluding multiple arrhythmia classes. The number of STE classes was the lowest at 185 . This dataset imbalance was present not only in the testing dataset but also in the training dataset. In the testing dataset, the arrhythmia group with the highest number of datasets was AF (260), and the arrhythmia group with the least number of datasets was STE (28). This dataset imbalance was also expected to affect the classification performance of the proposed model [23].

Accordingly, to prevent underestimation or overestimation of the proposed model due to dataset imbalance, the model was evaluated using the ROC curve, precision-recall curve, and weighted F1 score [23]. This is because the ROC does not consider the difference in the number of datasets between classes, while the precision-recall curve allows us to check the classification performance of the model taking into account the difference in the number of datasets $[23,24]$. In addition, when calculating the final F1 score, the model was objectively evaluated by multiplying the F1 score by the weight, taking into account the number of datasets for each class during the classification of multiclass data.

In this study, we proposed a clustering algorithm that can classify persistent cardiac arrhythmia as well as episodic cardiac arrhythmias using standard 12-lead ECG signals. The 2D CNN model also used timefrequency feature maps to classify the eight types of arrhythmias. In particular, the proposed algorithm showed excellent performance in the classification of persistent cardiac arrhythmias. The somewhat low accuracy in the classification of episodic arrhythmias may be due to dataset imbalance in the episodic arrhythmia group. If this problem is compensated for through learning and verification using more datasets for episodic arrhythmias, we believe our clustering algorithm can be used as an auxiliary device for diagnosing cardiac arrhythmias.

\section{Methods}




\section{1. Standard 12-leads ECG database}

In this study, the standard 12-leads ECG database provided by the Computing in Cardiology 2020 Physionet challenge was used; it consists of 6,877 datasets (male: 3,699 , female: 3,178 ) [25]. These datasets contain ECG signals obtained from eight types of cardiac arrhythmia patients (AF, I-AVB, LBBB, RBBB, PAC, PVC, STD, and STE patients), including NSR signals without any cardiac arrhythmias. Each signal was obtained at a sampling frequency of $500 \mathrm{~Hz}$, and each ECG signal was measured for a minimum of $6 \mathrm{~s}$ to a maximum of $60 \mathrm{~s}$.

\section{2. Preprocessing}

\section{2. 1. Removing motion artifact and baseline wandering}

Figure 1 shows the overall process used by the proposed artificial intelligence algorithm to classify the eight types of cardiac arrhythmias. First, a band-pass filter was applied to remove the signal noise and baseline wandering noise while taking measurements using an ECG device. In this study, we filtered the signals between 0.5 and $20 \mathrm{~Hz}$ to remove motion artifacts and minimize data loss based on the advanced study done by Stridth et al. [18].

\section{2. 2. R-R grouping algorithm}

The filtered 12-lead ECG signals were grouped based on the R-peaks detected from the filtered ECG signal of lead $\mathrm{I}$, which is the preparation process for generating the sequence signal that includes both the change in heart rate and shape deformation due to the arrhythmias, to classify the eight types of cardiac arrhythmias. The R-peak of each ECG signal was extracted by combining the Pan and Tompkins algorithm and a robust thresholding algorithm [26, 27].

\section{2. 3. Clustering algorithm for extracting representative signals}

A clustering algorithm for extracting representative signals including geometric information due to episodic arrhythmias is shown in the workflow diagram in Fig. 2b. First, continuous signal sequences, including three PQRST signals, were divided into two groups (A group and B group). To find a group of signals with abnormal signs due to a single cardiac arrhythmia, the representative signal is randomly extracted from the small groups when the ratio of the small group to the large group is 0.35 or less. If this ratio is greater than 0.35 , the representative signal is randomly extracted from the large group. If the number of groups is the same, clustering is performed again in each group, and each group is subdivided into four groups $\left(\mathrm{S}_{\mathrm{A} 1}, \mathrm{~S}_{\mathrm{A} 2}, \mathrm{~S}_{\mathrm{B} 1}, \mathrm{~S}_{\mathrm{B} 2}\right)$. The representative signal is then extracted from the smallest group. The extracted signal, which comprises abnormal signs due to episodic cardiac arrhythmias and persistent cardiac arrhythmias, was used as a representative signal $\left(\mathrm{S}_{\#}\right)$.

\section{2. 4. Time-frequency feature map extraction}


The representative signals extracted from each of the 12 channels were transformed into a timefrequency feature map through STFT. The ECG signals measured from the ECG machines generally contain information in the frequency band between 0 and $150 \mathrm{~Hz}$ [28]; however, in this study, we used only information in the frequency band between 0 and $50 \mathrm{~Hz}$ generated from STFT (frequency characteristics due to AF are captured in the $2.5-25 \mathrm{~Hz}$ frequency band [18]). The time-frequency feature maps extracted from the 12 ECG channels were sequentially merged and used (Fig. 2a). For the STFT, the frequency window was set to $300 \mathrm{~Hz}$ to observe the frequency change over time with a frequency resolution of $1.667 \mathrm{~Hz}$. The overlap ratio was set at $270 \mathrm{~Hz}$ to minimize the decrease in time resolution due to the increase in frequency resolution. Because the length of the R-R sequences differs according to the type of arrhythmia, the time-frequency maps were transformed into final time-frequency maps with a shape of $120 \times 120$, using linear interpolation [9] (Fig. 2b). Finally, these time-frequency maps were used as inputs to the $2 \mathrm{D} \mathrm{CNN}$ model.

\section{3. Model structure}

The CNN model for classifying cardiac arrhythmias consisted of three 2D CNN layers, a BatchNormalization (BN) layer, and a 2D Maxpooling (2DmaxP) layer (2DCNN-BN-2DmaxP). A dropout rate of 0.5 was applied to the first and second 2DCNN-BN-2DmaxP layers, and 0.3 for the last 2DCNN-BN2DmaxP layer. Kernel normalization and bias normalization with a loss lambda of 0.00015 were applied to each 2D CNN layer to prevent the model from overfitting with the training dataset. ReLU was used as the activation function for each layer, and "softmax" was used as the activation function for the output layer [29].

$80 \%$ of the dataset was used for training $(5,484)$ and $20 \%$ for testing $(1,372)$. The model was trained 500 times using the sparse categorical cross-entropy loss function and the Adam optimization function with a learning rate of 0.01 [30], and the optimal model was verified using 5 -fold cross-validation. The classification performance of the final model was evaluated in terms of accuracy, recall, and precision. The F1 score, confusion matrix, ROC curve, and precision-recall curve were also evaluated [24].

\section{Declarations}

\section{Acknowledgments}

This research was partially supported by the NRF (National Research Foundation) under basic engineering research project (2016R1D1A1B0101440), the EDISON (NRF-2011-0020576) Programs, and the Grand Information Technology Research Center Program through the Institute of Information \& Communications Technology and Planning \& Evaluation (IITP) funded by the Ministry of Science and ICT (MSIT), Korea (IITP-2021-2020-0-01612).

\section{Author contributions}

This manuscript is an intellectual product of the entire team. DUJ wrote the machine learning source code Loading [MathJax]/jax/output/CommonHTML/jax.js hd interpreted the results. KML designed the study and 
reviewed and revised the entire manuscript based on the results. All authors have read and approved the final manuscript.

\section{Additional information}

\section{Data availability}

The standard 12-lead ECG dataset used in this study can be downloaded from the Physionet website for Classification of 12-lead ECGs: the PhysionNet - Computing in Cardiology Challenge 2020; https://physionet.org/content/challenge-2020/1.0.1/.

All results were generated through machine learning performed by the authors based on the methods described in the text.

\section{Competing interests}

The authors declare that the study was conducted in the absence of any commercial or financial relationship that could be construed as a potential conflict of interest.

\section{References}

1. Rajendra Acharya, U., Suri, J. S., Spaan, J. A. E. \& Krishnan, S. M. Advances in cardiac signal processing. Advances in Cardiac Signal Processing (2007). doi:10.1007/978-3-540-36675-1.

2. Ashley, E. A. \& Niebauer, J. Cardiology explained. (Andrew Ward, 2004).

3. Hagiwara, Y. et al. Computer-aided diagnosis of atrial fibrillation based on ECG Signals: A review. Inf. Sci. (Ny).467, 99-114 (2018).

4. Uhm, J.-S. et al. First-degree atrioventricular block is associated with advanced atrioventricular block, atrial fibrillation and left ventricular dysfunction in patients with hypertension. J. Hypertens.32, 1115-1120 (2014).

5. Dale, D. Cardiology - Rapid Interpretation of EKG's. (cover publishing company, 2000).

6. Fred, K. ECG Interpretation: From Pathophysiology to Clinical Application. Springer Nature vol. (5)2 (Springer Nature, 2020).

7. Krasteva, V., Jekova, I. \& Christov, I. Automatic detection of premature atrial contractions in the electrocardiogram. Clbme.Bas.Bg 9-10 (2006).

8. Inan, O. T., Giovangrandi, L. \& Kovacs, G. T. A. Robust neural-network-based classification of premature ventricular contractions using wavelet transform and timing interval features. IEEE Trans. Biomed. Eng.53, 2507-2515 (2006).

9. Ullah, A., Anwar, S. M., Bilal, M. \& Mehmood, R. M. Classification of arrhythmia by using deep learning with 2-D ECG spectral image representation. Remote Sens.12, 1-14 (2020).

10. Shi, L., Yang, C., Zhang, J. \& Li, H. Recognition of ST segment of electrocardiogram based on wavelet 
11. Datta, S. et al. Identifying normal, AF and other abnormal ECG rhythms using a cascaded binary classifier. Comput. Cardiol. (2010).44, 1-4 (2017).

12. Dohare, A. K., Kumar, V. \& Kumar, R. Detection of myocardial infarction in 12 lead ECG using support vector machine. Appl. Soft Comput. J.64, 138-147 (2018).

13. Baloglu, U. B., Talo, M., Yildirim, O., Tan, R. S. \& Acharya, U. R. Classification of myocardial infarction with multi-lead ECG signals and deep CNN. Pattern Recognit. Lett.122, 23-30 (2019).

14. Ribeiro, A. H. et al. Automatic diagnosis of the 12-lead ECG using a deep neural network. Nat. Commun.11, 1-9 (2020).

15. Mostayed, A., Luo, J., Shu, X. \& Wee, W. Classification of 12-Lead ECG signals with bi-directional LSTM network. arXiv 1-16 (2018).

16. Miwakeichi, F. et al. Decomposing EEG data into space-time-frequency components using Parallel Factor Analysis. Neuroimage22, 1035-1045 (2004).

17. Hurnanen, T. et al. Automated Detection of Atrial Fibrillation Based on Time-Frequency Analysis of Seismocardiograms. IEEE J. Biomed. Heal. Informatics21, 1233-1241 (2017).

18. Stridh, M., Sörnmo, L., Meurling, C. J. \& Olsson, S. B. Sequential Characterization of Atrial Tachyarrhythmias Based on ECG Time-Frequency Analysis. IEEE Trans. Biomed. Eng.51, 100-114 (2004).

19. Chun, S. Y. et al. ECG based user authentication for wearable devices using short time Fourier transform. 2016 39th Int. Conf. Telecommun. Signal Process. TSP 2016 656-659 (2016) doi:10.1109/TSP.2016.7760964.

20. Ng, J. \& Goldberger, J. J. Understanding and interpreting dominant frequency analysis of AF electrograms. J. Cardiovasc. Electrophysiol.18, 680-685 (2007).

21. Benmalek, E. \& Elmhamdi, J. Arrhythmia ECG signal analysis using non parametric time-frequency technique. Proc. 2015 Int. Conf. Electr. Inf. Technol. ICEIT 2015 281-285 (2015) doi:10.1109/EITech.2015.7162958.

22. Classification of 12-lead ECGs: the PhysioNet/Computing in Cardiology Challenge 2020. Computers in Cardiology https://physionetchallenges.org/ (2020).

23. Jeni, L. A., Cohn, J. F. \& De La Torre, F. Facing imbalanced data - Recommendations for the use of performance metrics. Proc. - 2013 Hum. Assoc. Conf. Affect. Comput. Intell. Interact. ACII $2013245-$ 251 (2013) doi:10.1109/ACII.2013.47.

24. Fawcett, T. An introduction to ROC analysis. Pattern Recognit. Lett.27, 861-874 (2006).

25. Classification of 12-lead ECGs: the PhysioNet/Computing in Cardiology Challenge 2020. 2020 Computing in Cardiology (CinC) https://physionetchallenges.github.io/2020/ https://physionetchallenges.github.io/2020/ (2020).

26. Pan, J. \& Tompkins, W. J. A Real-Time QRS Detection Algorithm. IEEE Trans. Biomed. Eng.BME-32, 230-236 (1985). 
27. Kathirvel, P., Manikandan, M. S., Prasanna, S. R. M. \& Soman, K. P. An Efficient R-peak Detection Based on New Nonlinear Transformation and First-Order Gaussian Differentiator. Cardiovasc. Eng. Technol.2, 408-425 (2011).

28. Tereshchenko, L. G. \& Josephson, M. E. Frequency Content and Characteristics of Ventricular Conduction. J. Electrocardiol.48, 933-937 (2015).

29. Xavier, G., Antoine, B. \& Yoshua, B. Deep sparse rectifier neural networks. in International Conference on Artificial Intelligence and statistics (AISTATS) vol. 15 315-323 (2011).

30. Kingma, D. P. \& Ba, J. L. Adam: A method for stochastic optimization. in 3rd International Conference on Learning Representations, ICLR 2015 - Conference Track Proceedings 1-15 (2015).

\section{Figures}

(a)

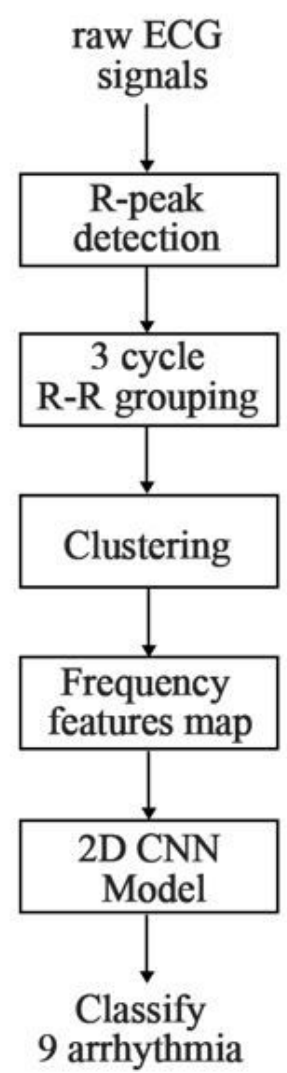

(b)

3 cycle R-R grouped signals

Clustering into 2 groups

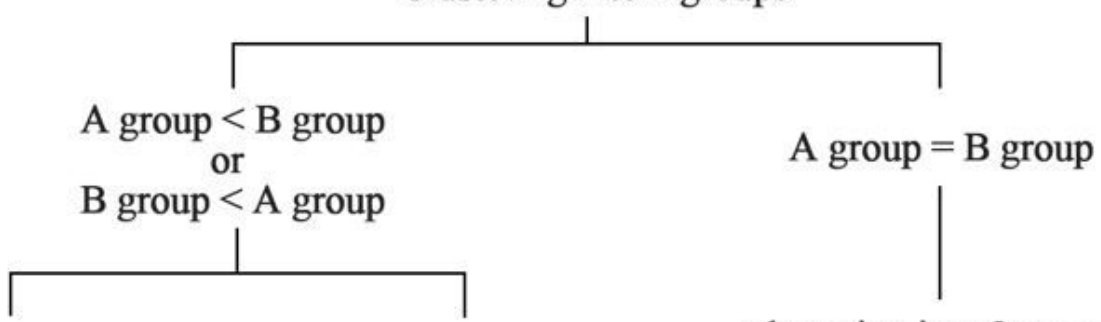

clusetring into 2 groups

small group $\leq 0.35 \quad$ small group $>0.35$

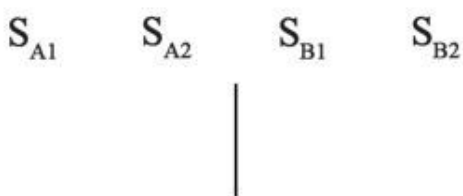

Randomly choose $\mathrm{s}_{\mathrm{i}}$ from large group

Randomly choose

Randomly choose
$\mathbf{S}_{\| \text {fit }}$ from small group

$\mathrm{S}_{\#}$ : representative signal

\section{Figure 1}

The workflow of the proposed model. (a) The workflow for the entire process; (b) the workflow for the clustering algorithm. 
(a)

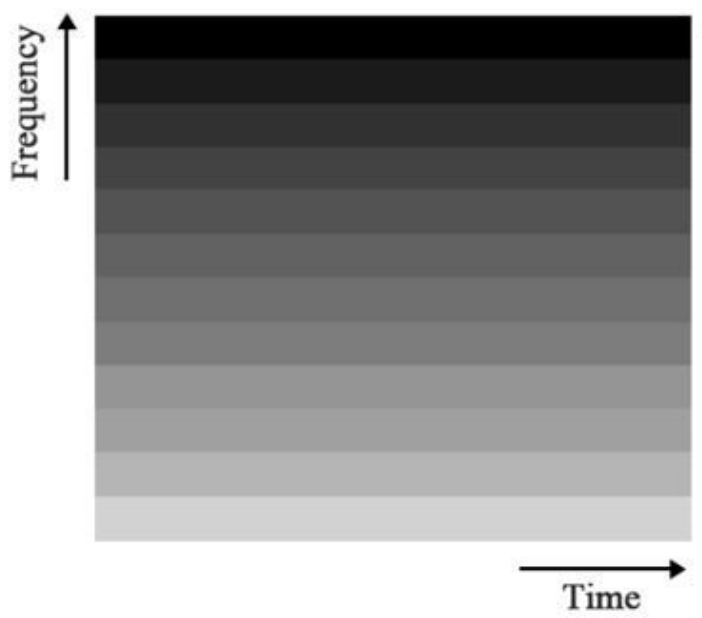

(b)

V6

V5

V4

V3

V2

V1

aVL

aVF

aVR

Lead III

Lead II

Lead I

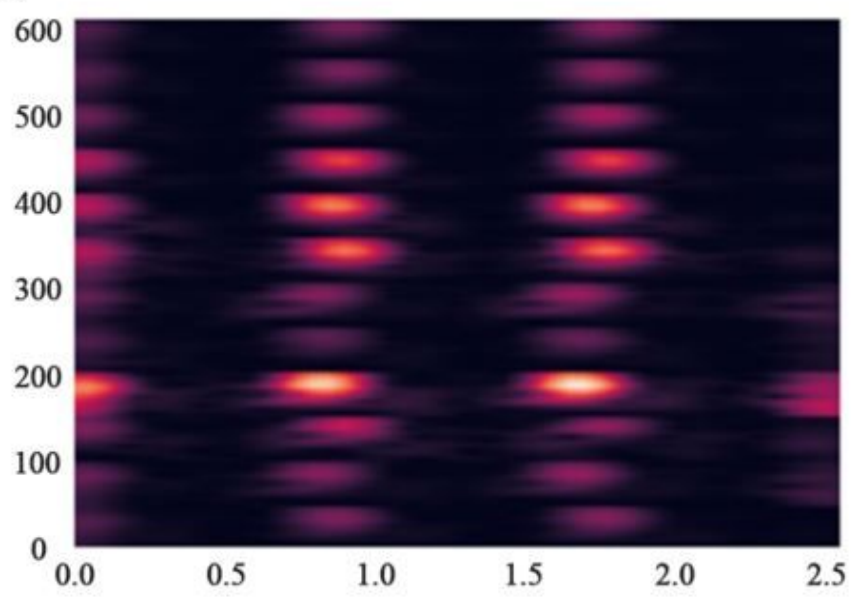

Figure 2

Time-frequency feature map. (a) Mimetic diagram of the time-frequency feature map; (b) a timefrequency feature map generated from the proposed algorithm. 


\section{Confusion matrix}

\begin{tabular}{|c|c|c|c|c|c|c|c|c|c|}
\hline $\mathrm{AF}$ & 215 & 15 & 1 & 0 & 7 & 4 & 15 & 1 & 2 \\
\hline I-AVB & 2 & 118 & 0 & 4 & 0 & 4 & 9 & 2 & 2 \\
\hline LBBB & 0 & 2 & 33 & 0 & 0 & 2 & 0 & 1 & 2 \\
\hline Normal & 0 & 5 & 0 & 150 & 4 & 5 & 6 & 17 & 5 \\
\hline PAC & 8 & 4 & 0 & 9 & 50 & 12 & 7 & 15 & 1 \\
\hline PVC & 7 & 3 & 0 & 1 & 10 & 68 & 13 & 7 & 2 \\
\hline $\mathrm{RBBB}$ & 7 & 6 & 0 & 11 & 7 & 6 & 294 & 8 & 3 \\
\hline STD & 0 & 0 & 0 & 18 & 4 & 1 & 4 & 125 & 0 \\
\hline STE & 1 & 0 & 0 & 6 & 1 & 1 & 2 & 1 & 16 \\
\hline
\end{tabular}

Figure 3

Confusion matrix for the proposed model

(a)

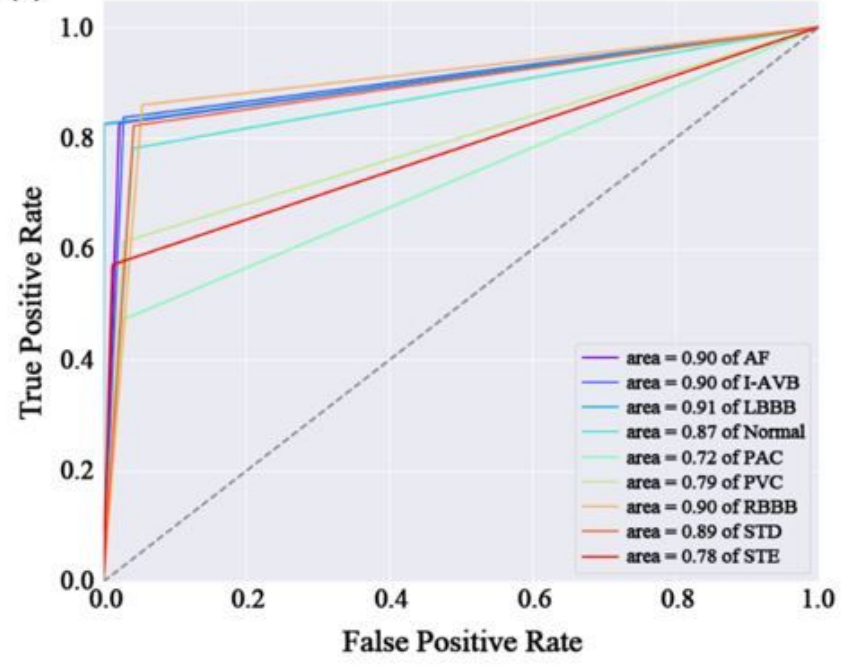

(b)

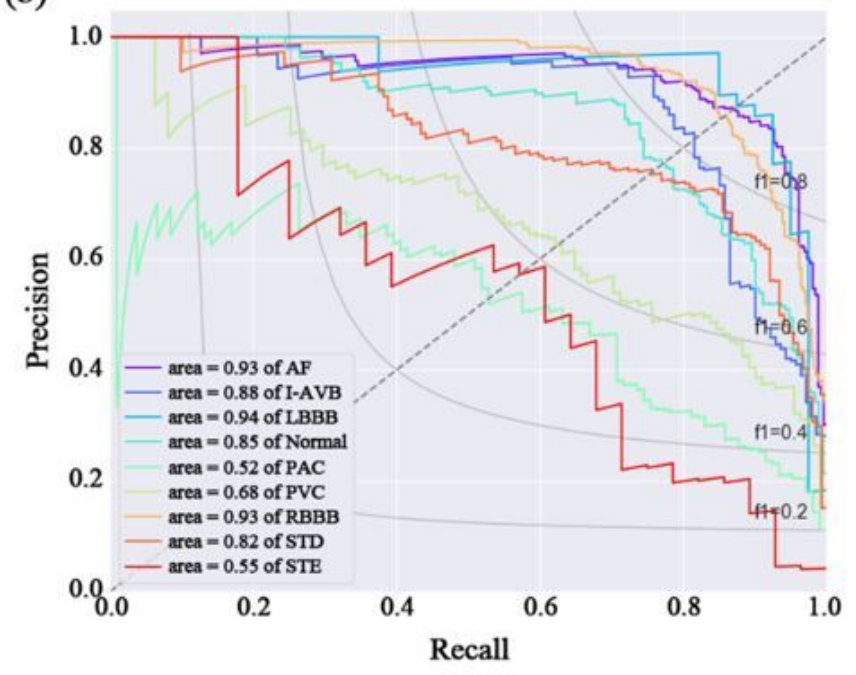


Figure 4

Performance curves for the proposed model. (a) Receiver operating characteristics (ROC) curves; (b) Precision-recall curves.

\section{Supplementary Files}

This is a list of supplementary files associated with this preprint. Click to download.

- OSupplementaryECG.pdf 